\title{
Creatine and Alpha-Lipoic Acid Antidepressant-Like Effect Following Cyclosporine A Administration
}

\author{
(1) Mehdi ALIOMRANI', (1) Azadeh MESRIPOUR2*, (1) Abolfazl Saleki MEHRJARDI \\ 1Isfahan University of Medical Sciences, School of Pharmacy and Pharmaceutical Sciences, Department of Pharmacology and Toxicology, Isfahan, Iran \\ 2Isfahan University of Medical Sciences, School of Pharmacy and Pharmaceutical Sciences, Isfahan Pharmaceutical Sciences Research Center, Isfahan, \\ Iran
}

\begin{abstract}
Objectives: Cyclosporine A (CYA), is an immunosuppressant drug used to prevent graft rejection, but it may initiate neuropsychological problems such as depression. The aim was to evaluate the antidepressant-like effects of creatine (Crt), a mediator of oxidative phosphorylation, and alphalipoic acid (ALA), a cofactor for the mitochondrial respiratory chain following CYA administration.

Materials and Methods: Female mice ( $27 \pm 2 \mathrm{~g}$ ) were used, immobility time during the forced swimming test (FST) was measured, and hippocampal brain-derived neurotrophic factor (BDNF) level was evaluated. CYA $20 \mathrm{mg} / \mathrm{kg}$, ALA $40 \mathrm{mg} / \mathrm{kg}$, fluoxetine $20 \mathrm{mg} / \mathrm{kg}$, and Crt $10 \mathrm{mg} / \mathrm{kg}$ (oral) were administered for 6 consecutive days, and the tests were performed on day 7 .

Results: ALA, but not Crt, treatment alone decreased immobility in the FST (i.e., decreases depression-like behavior). CYA administration increased immobility in the FST $(175.1 \pm 13.16 \mathrm{~s}$, vs. vehicle $130.9 \pm 13.5 \mathrm{~s}, p=0.0364)$, and this depression-like behavior was prevented by co-administrating, ALA (100 $\pm 15.9 \mathrm{~s}, p=0.020)$ or Crt $(93.5 \pm 16.6, p=0.009)$ and the positive control, fluoxetine. Notably, there was a synergistic effect of Crt-ALA coadministration since CYA-induced immobility was lower in this group than in the groups pretreated with Crt or ALA. These behavioral changes were observed without treatment effects on locomotor activity in an open field. CYA treatment increased hippocampal BDNF protein levels prevented by co-administration of ALA (with or without $\mathrm{Crt}$ ) or fluoxetine.

Conclusion: CYA-induced depression-like behavior might be related to hippocampal mitochondrial dysfunction as ALA and Crt prevented the development of this behavioral phenotype. ALA, similar to fluoxetine, prevented BDNF alteration and its possible neurological changes.
\end{abstract}

Key words: Cyclosporine, depression, creatine, alpha-lipoic acid, brain-derived neurotrophic factor

\section{INTRODUCTION}

Calcineurin, a calcium-dependent protein phosphatase enzyme, is well known for its effect as a modulator of the immune response. It also participates in neurotransmission, neuronal structure, and neuronal excitabilit.1.2 Calcineurin inhibiting drugs, such as cyclosporine A (CYA), are immunosuppressive drugs that have developed the organ transplantation process by extensively reducing allograft rejection rates in individuals.,4 However, some patients that receive these agents suffer from neuropsychological problems such as depression, anxiety, confusion, and tremor. ${ }^{5}$ Peripheral administration of high dose $(60 \mathrm{mg} / \mathrm{kg}$ ) CYA has decreased the release of serotonin and dopamine and caused prefrontal cortex dysfunction that could be responsible for the increased anxiety and social behavior disturbance. ${ }^{6}$ Additionally, CYA may induce neurotoxicity by interaction with brain mitochondria functioning. ${ }^{5}$ Brain-derived neurotrophic factor (BDNF) belongs to the neurotrophin family; it is the most plentiful neurotrophin in the central nervous system (CNS) and is related to neural cell survival and neural transmission. ${ }^{7}$ Reduction of BDNF expression in hippocampal neurons would cause severe stress and influence learning, inspiration, and mood. 8,9

Creatine ( $\mathrm{N}$-aminoiminomethyl- $\mathrm{N}$-methylglycine; $\mathrm{Crt}$ ) can be endogenously synthesized by the liver, kidney, pancreas, and to some extent in the brain from the amino acids arginine, glycine, and methionine. $\mathrm{Crt}$ is also provided in diets having meat or fish. ${ }^{10}$ Adenosine triphosphate (ATP) is the primary energy source in the brain that is closely joined to phosphocreatine

*Correspondence: a_mesripour@yahoo.com, Phone: +0989131000614, ORCID-ID: orcid.org/0000-0003-3150-5581

Received: 27.06.2021, Accepted: 16.08.2021

๑Turk J Pharm Sci, Published by Galenos Publishing House. 
$(\mathrm{PC} r$ ). The isoenzymes of $\mathrm{Crt}$ kinase are specially localized in high-demanding ATP sites like the neurons to regenerate ATP in situ via $\mathrm{PCr} .^{10}$ Therefore, oxidative phosphorylation and $\mathrm{Crt} / \mathrm{PCr}$ system prepare high energy that is critical for CNS function.1 $\mathrm{Crt}$ has various properties in the CNS, including antioxidant, antiinflammatory, anti-apoptotic, and neuromodulatory activity.10,12 These effects have provoked further research regarding $\mathrm{Crt}$ monohydrate efficacy for treating neurological disorders.

Alpha-lipoic acid (ALA) naturally occurs in vegetables like broccoli, spinach, and tomatoes. ${ }^{13}$ ALA is an essential cofactor for mitochondrial respiratory chain enzymes $\alpha$-ketodehydrogenase complexes..$^{14} \mathrm{ALA}$ and its reduced metabolite dihydrolipoic acid have been noticed as antioxidants against hydroxyl radicals and an inhibitor of lipid and protein oxidation. Interestingly, this free-radical quenching antioxidant, in contrast to vitamin $E$ (fat-soluble), is soluble in both fat and water; thus, it deactivates free radicals in both fatty and watery areas of cells. ALA can readily spread into CNS and induce protective effects on the nervous system, leading to its promising therapeutic effects on brain disorders. ${ }^{14}$

Since CYA might induce depression side effects and administrating prophylactic antidepressant drugs would expose the individual to unnecessary medication and polypharmacy, the aim was to introduce an alternative medicine. Therefore, treatment with two supplements, ALA (cofactor for mitochondrial respiratory chain) and Crt (mediator of mitochondria oxidative phosphorylation), was evaluated on mice's CYA-induced behavior changes. Novelty of the study was that the antidepressant effects of $\mathrm{Crt}$ and ALA were evaluated following CYA administration in mice, and finally, the BDNF levels were assessed in mice brains.

\section{MATERIALS AND METHODS}

\section{Animals}

Female NMRI mice (weighing $27 \pm 2$ g, 6-8 weeks old) were housed six in each cage and kept at room temperature 21 $\pm 2^{\circ} \mathrm{C}$ on a $12 \mathrm{~h}$ light and $12 \mathrm{~h}$ dark cycle (lights on at 06:00 AM); standard mice chow and tap water ad libitum. Cages were placed in the behavioral laboratory $24 \mathrm{~h}$ prior to the experiments to acclimatize. The experiments were carried out according to the Care and Use of Laboratory Animals Guidelines Issued by The National Ethical Committee of Vice-Chancellor in Research Affairs-Medical University of Isfahan (ethical no: IR.MUI.RESEARCH.REC.1399.200). All the attempts were made to reduce animal distress and the number of animals used in the research.

\section{Drug administration}

CYA (Sandimmun, 50 mg/mL; Novartis, Switzerland) 20 mg/ $\mathrm{kg}$ was injected intraperitoneal (IP) after diluted in $2 \% \mathrm{v} / \mathrm{v}$ $\mathrm{EtOH} /$ normal saline. ${ }^{15} \mathrm{Crt}$ monohydrate (Karen Pharma and Food Supplement, Iran) $10 \mathrm{mg} / \mathrm{kg}$ was administered by daily gavage feeding tube, ${ }^{16}$ ALA (Sigma Aldrich, India) $40 \mathrm{mg} / \mathrm{kg}$ was injected IP.17 A selective serotonin reuptake inhibitors (SSRIs) fluoxetine (Sigma-Aldrich, Germany) 20 mg/kg was injected IP. ${ }^{15}$ Control groups were injected with normal saline or received normal saline by gavage feeding tube. In a separate group of mice, $\mathrm{Crt}$ and ALA were co-administration with CYA. The volume for all of the injections was $10 \mathrm{~mL} / \mathrm{kg}$.

\section{Experiment design}

Totally 9 groups of animals consisting of 6 mice in each group were studied. Groups included: groups that received each of the drugs ( $\mathrm{ALA}, \mathrm{Crt}$ ) alone and the control group that received normal saline (data for IP injection and gavage were similar; therefore, one group was considered here). The CYA alone group and the vehicle group ( $2 \% \mathrm{v} / \mathrm{v} \mathrm{EtOH} /$ normal saline). Three groups that received ALA, Crt or fluoxetine (the positive control) concomitantly with CYA; finally, a group that was treated with $\mathrm{ALA}+\mathrm{Crt}$ together with CYA. All the treatments were administered for 6 consecutive days, and the tests were performed on day 7 . The locomotor and forced swimming tests (FST) for measuring animal despair behavior were performed on each animal. After the animals were decapitated, the brain was carefully removed on ice and stored in -70 for BDNF evaluation.

\section{Locomotor test}

The locomotor activity of mice was assessed in an open arena $(45 \times 45 \mathrm{~cm})$ (Borj Sanat, Iran) divided into 15 zones by red beams. Mice were allowed to explore the field for $3 \mathrm{~min}^{; 18,19}$ by passing through the beams, the number of zone entries (horizontal exploration) was counted automatically, while rears on back-legs (vertical exploration) were recorded manually. The total activity for each animal was calculated, which was the sum of zone entries and rears on the back legs.

\section{Forced swimming test}

During FST, mice were forced to swim in $25^{\circ} \mathrm{C}$ water in a 2-liter Pyrex beaker (diameter $12.5 \mathrm{~cm}$, depth $12 \mathrm{~cm}$ ) for $6 \mathrm{~min}$; the first 2 min was considered for habituation. ${ }^{20}$ The animals' despair behavior was evaluated by measuring the immobility time during the last $4 \mathrm{~min}$ of the trial. It was considered when animals had no additional activity required to keep the animals' heads above the water. Swimming behavior, defined as horizontal movement throughout the beaker, which involved at least two limbs, and climbing behavior, defined as upward movements of the forepaws along the side of the beaker, was also recorded. The entire experiment was recorded by a camera and analyzed later. After $6 \mathrm{~min}$, the mice were dried carefully to avoid hypothermia and returned to their home cage.

\section{Brain BDNF enzyme-linked immunosorbent assay (ELISA)}

Mice were rapidly decapitated after $\mathrm{CO}_{2}$ euthanasia, and the whole brain was quickly removed, weighed, and the hippocampus was dissected on a cold tile. After that, the frozen samples were kept at $-70^{\circ} \mathrm{C}$ until further assay. Hippocampus of each sample was homogenized for $30 \mathrm{sec}$ with ice-cold extraction buffer containing $50 \mathrm{mM}$ Tris- $\mathrm{HCl}, 0.6 \mathrm{M} \mathrm{NaCl}, 0.2 \%$ Triton-X 100, 1\% bovine serum albumin, $0.1 \mathrm{mM}$ benzethonium chloride, $1 \mathrm{mM}$ benzamidine, and $0.1 \mathrm{mM}$ phenylmethylsulfonyl fluoride at $\mathrm{pH}$ 7.4. According to the manufacturer's instructions, a mice BDNF ELISA kit (Picokine TM ELISA, catalog no: 
EK0309, Boster Biological Technology, Canada) was used. Briefly, the homogenates were centrifuged $(10.000 \times g, 25 \mathrm{~min}$, $4^{\circ} \mathrm{C}$ ), and the supernatant was separated. ${ }^{21}$ After dilution, 100 $\mu \mathrm{L}$ of samples were added to each empty well and incubated at $37^{\circ} \mathrm{C}$ for 90 minutes. After washing the wells, $100 \mu \mathrm{L}$ of biotinylated anti-mice BDNF antibody working solution was added and incubated at $37^{\circ} \mathrm{C}$ for 60 minutes. Then all of the wells were washed three times with poly(butylene succinate) and incubated with avidin-biotin-peroxidase complex working solution at $37^{\circ} \mathrm{C}$ for 30 minutes. Finally, TMB color was added to each well and kept in the dark for 30 minutes. After changing the color to yellow following TMB stop solution addition, the plate optical density absorbance was read at $450 \mathrm{~nm}$ using a Synergy HTX microplate reader (BioTek, USA). All of the tests were run in duplicate. The standard curves ranged from 9.5 to $380 \mathrm{pg} / \mathrm{mL}$ BDNF with a $4 \mathrm{pg} / \mathrm{mL}$ sensitivity. The intra-assay coefficient of variation varied from 2.5 to $5.1 \%$, and the interassay coefficient of variation varied from 4.8 to $6.3 \%$. BDNF levels were calculated as $\mathrm{pg} / \mathrm{mg}$ of total protein.

\section{Statistical analysis}

Results were expressed as group mean \pm standard error of the mean. All behavior results were analyzed by One-Way ANOVA, followed by Tukey's multiple comparison tests. BDNF results were analyzed by One-Way ANOVA, followed by Tukey's multiple comparison tests. $P$ values less than 0.05 were considered significant. Excel 2010 and GraphPad Prizm (version 8) used the software programs for data analysis and making graphs.

\section{RESULTS}

\section{Effect of Crt, ALA, and CYA on the behavioral tests}

The locomotor activity results in Figure 1a show no statistically important difference in the total activity between $\mathrm{Crt}$ and ALA alone groups compared with the control group $[F(2,15)=3.544$, $p=1.724]$. Also, there was no significant difference in the total activity between CYA and the pretreatments with Crt, ALA, or fluoxetine groups compared with the vehicle group [F ( 5 , $30)=1.445, p=2.055]$. The results of immobility time during FST are presented in Figure 1b; $\mathrm{Crt}$ insignificantly reduced immobility time in normal animals while ALA significantly reduced immobility time $(81.6 \pm 15.9 \mathrm{~s} v \mathrm{~s}$. control $142 \pm 9.7 \mathrm{~s}, p=0.0094)$. CYA significantly increased immobility time $(175.1 \pm 13.16 \mathrm{~s}$, vs. vehicle $130.9 \pm 13.5 \mathrm{~s}, p=0.0364)$, and by pretreatments, with ALA (100 $\pm 15.9 \mathrm{~s}, p=0.020)$ or $\operatorname{Crt}(93.5 \pm 16.6, p=0.009)$ the immobility time significantly decreased compared to CYA alone. By co-administration of ALA-Crt following CYA injection, immobility time dropped dramatically $(12.2 \pm 2.59 \mathrm{~s}, p<0.001 \mathrm{vs}$. CYA). The result was significantly lower than pretreatment with ALA ( $p=0.0022)$ or Crt separately ( $p=0.0052)$. Fluoxetine as the reference antidepressant drug reduced immobility in CYAtreated animals ( $47.7 \pm 18.8 \mathrm{~s}, p<0.001 \mathrm{vs.}$. CYA group).

According to Table 1, swimming time during the FST following ALA administration was significantly higher than control (141 \pm $13.9 \mathrm{~s} v$ s. control $76.9 \pm 11 \mathrm{~s}, p=0.002$ ) Crt also slightly increased swimming time. By administrating $\mathrm{Crt}$ and $\mathrm{CYA}$, swimming time was significantly higher $(p=0.0393)$ than CYA alone.
Similarly, co-administrating ALA-Crt increased swimming time compared to CYA alone $(p=0.0030$ ). The table also shows that treating with ALA-Crt significantly increased the climbing time compared to CYA alone ( $p=0.0298$ ). Climbing time was also significantly higher in animals treated with fluoxetine than CYA alone $(p<0.001)$.

\section{Brain BDNF level}

The results of brain BDNF levels are depicted in Figure 2. BDNF level was not different following administrating ALA or Crt compared to the control group $(571 \pm 43.1 \mathrm{pg} / \mathrm{mg}$ protein). Interestingly CYA significantly increased BDNF levels compared with vehicle $(1059 \pm 81.0 \mathrm{pg} / \mathrm{mg}$ protein, $p=0.0093$ ) Treatment with $\mathrm{Crt}$ did not change the result, while treatment with ALA similar to fluoxetine significantly reduced BDNF levels compared with CYA alone $(684 \pm 103 \mathrm{pg} / \mathrm{mg}$ protein, $p=$ 0.0454). Administrating ALA-Crt also significantly decreased BDNF levels compared with CYA alone ( $p=0.0413$ ). Table 2

a)

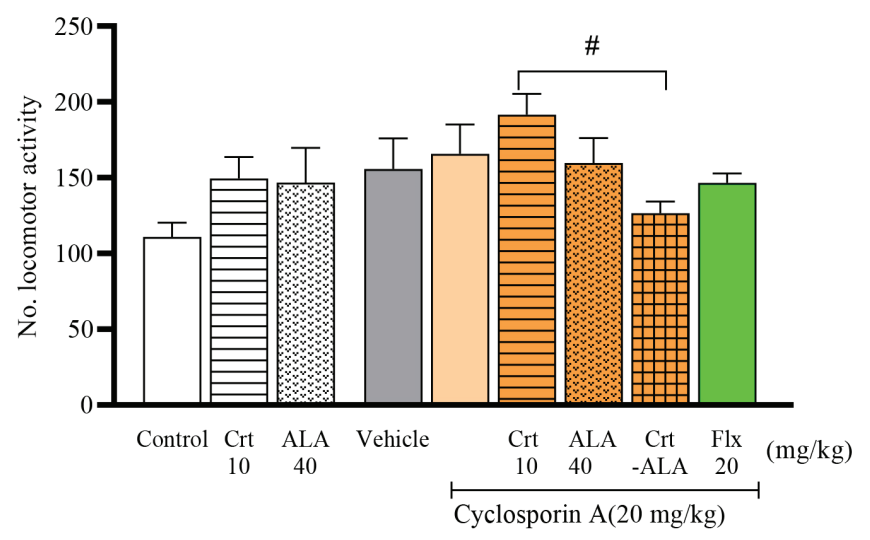

b)

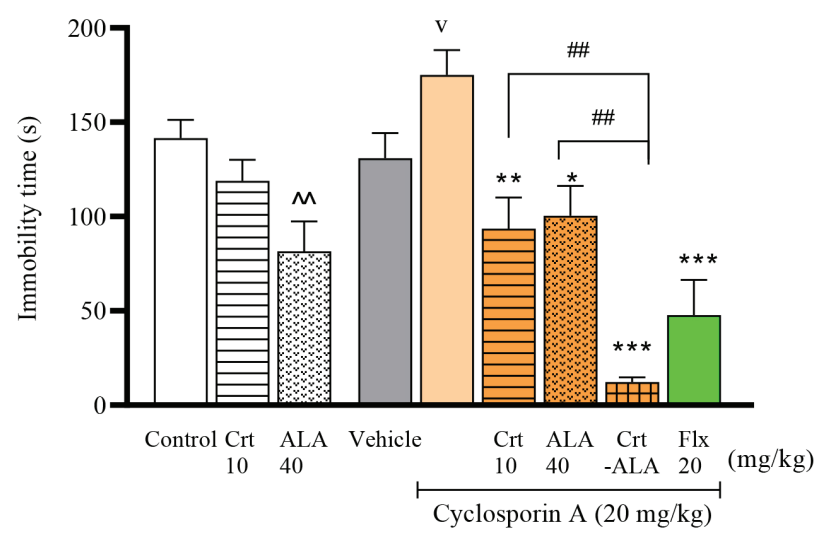

Figure 1. Effect of $\mathrm{Crt}$, and $\mathrm{ALA}$, on mice behavior following CYA administration. Total activity during locomotor test $=$ (horizontal + vertical) exploration (a), and immobility time during FST (b). The control and vehicle groups received normal saline and $2 \% \mathrm{v} / \mathrm{v} \mathrm{EtOH} / \mathrm{normal}$ saline. Results are expressed as group mean \pm SEM and analyzed by ANOVA followed by Tukey's comparison test $(n=6){ }^{\wedge} p<0.05$, compared with the control; ${ }^{v} p<0.05$, compared with the vehicle. ${ }^{*} p<0.05,{ }^{* *} p<0.01$, and ${ }^{* * *} p<0.001$ compared with CYA group. \#\#p<0.01 as shown

ALA: Alpha-lipoic acid, Crt: Creatine, CYA: Cyclosporine A, SEM: Standard error of the mean 
Table 1. Swimming and climbing time during the FST

\begin{tabular}{lll} 
Groups $(n=6)$ & $\begin{array}{l}\text { Swimming } \\
\text { time }(s)\end{array}$ & $\begin{array}{l}\text { Climbing time } \\
(\mathrm{s})\end{array}$ \\
\hline Control & $76.9 \pm 11$ & $21.14 \pm 12.01$ \\
\hline ALA & $141 \pm 13.9^{\wedge}$ & $17.50 \pm 10.16$ \\
\hline Crt & $113 \pm 9.65$ & $8.50 \pm 3.26$ \\
\hline Vehicle & $76.3 \pm 17.4$ & $1.66 \pm 0.91$ \\
\hline CYA & $69.7 \pm 18.3$ & $3.16 \pm 1.68$ \\
\hline CYA+ALA & $117 \pm 10.1$ & $22.50 \pm 9.60$ \\
\hline CYA+Crt & $132 \pm 18.2^{\star}$ & $20.17 \pm 8.64$ \\
\hline CYA+ALA+Crt & $174 \pm 18.1^{* *, v v}$ & $54.17 \pm 18.67^{\star}$ \\
\hline CYA+fluoxetine & $84.2 \pm 21.2$ & $91.50 \pm 15.51^{\star * *}$ \\
\hline
\end{tabular}

The control and vehicle groups received normal saline and $2 \% \mathrm{v} / \mathrm{v} \mathrm{EtOH/}$ normal saline. Results are expressed as group mean \pm SEM and analyzed by ANOVA followed by Tukey's comparison test $(n=6){ }^{\wedge}{ }^{\wedge}: p<0.05$, compared with the control; vv: $p<0.01$, compared with the vehicle. ${ }^{*}: p<0.05,{ }^{* *}: p<0.01$, compared with CYA group. FST: Forced swimming test, ALA: Alpha-lipoic acid, Crt: Creatine, CYA: Cyclosporine A, SEM: Standard error of the mean

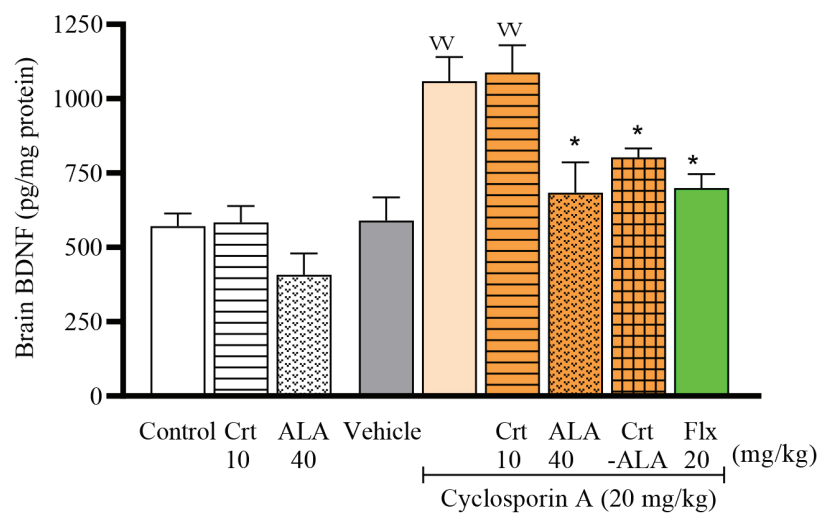

Figure 2. Normalized brain-derived BDNF level. The control and vehicle groups received normal saline and $2 \% \mathrm{v} / \mathrm{v} \mathrm{EtOH/normal} \mathrm{saline.} \mathrm{Results}$ are expressed as group mean \pm SEM and analyzed by ANOVA followed by Tukey's comparison test $(n=6)$. "v $p<0.01$, compared with the vehicle; ${ }^{*} p<0.05$, compared with the CYA group

BDNF: Brain-derived neurotrophic factor, SEM: Standard error of the mean, CYA: Cyclosporine A, ALA: Alpha-lipoic acid, Crt: Creatine

shows the percentage of the hippocampus to whole-brain tissue; in CYA treatments, it was significantly higher than the vehicle group, and $\mathrm{ALA}$ and $\mathrm{Crt}$ reversed it, but not fluoxetine.

\section{DISCUSSION}

This research proved that the complementary drugs, ALA and Crt, prevent depressive-like behavior initiation by CYA in mice, and ALA-Crt showed a synergistic antidepressant effect. While CYA increased the brain BDNF level, treatment with Crt did not change the BDNF level, and ALA reduced it. This study was carried out on female mice since it was shown previously that Crt effects on depressive behavior in FST are sex-dependent, and female rats had shown better antidepressant-like response. $^{22}$
Table 2. Hippocampus to whole-brain percentage

\begin{tabular}{ll} 
Groups $(n=5)$ & $\begin{array}{l}\% \\
\text { Hippocampus } \\
\text { to the whole } \\
\text { brain }\end{array}$ \\
\hline Control & $9.02 \pm 0.44$ \\
\hline ALA & $7.15 \pm 1.22$ \\
\hline Crt & $6.31 \pm 0.52^{\wedge}$ \\
\hline Vehicle & $8.06 \pm 0.32$ \\
\hline CYA & $10.34 \pm 0.54^{\vee}$ \\
\hline CYA+ALA & $7.42 \pm 0.98^{\star}$ \\
\hline CYA+Crt & $8.58 \pm 0.51$ \\
\hline CYA+ALA+Crt & $8.22 \pm 0.76$ \\
\hline CYA+fluoxetine & $11.31 \pm 0.31^{\vee}$ \\
\hline
\end{tabular}

The control and vehicle groups received normal saline and $2 \% \mathrm{v} / \mathrm{v} \mathrm{EtOH/}$ normal saline. Results are expressed as group mean \pm SEM and analyzed by ANOVA followed by Tukey's comparison test $(n=6){ }^{\wedge}$ : $p<0.05$, compared with the control; ${ }^{v}: p<0.05$, compared with vehicle; ${ }^{*} p<0.05$, compared with the CYA group. ALA: Alpha-lipoic acid, Crt: Creatine, CYA: Cyclosporine A, SEM: Standard error of the mean

FST is the most commonly used behavior test used for antidepressant screening. ${ }^{23}$ Rodents perform a typical immobile posture following initial escape attempts movements in the inescapable water-filled beaker. Immobility time during FST was measured as a certain depressive-like phenotype that is despair behavior. The locomotor activity was evaluated prior to the FST since no noticeable difference was observed between different therapies and normal animals; thus, changes in the immobility time in FST could be interpreted as animal depressive-like behavior. CYA administration increased immobility time, indicating animal despair behavior. It was also reported previously that following CYA single-dose injection, immobility time measured after $4 \mathrm{~h}$ and $24 \mathrm{~h}$ in separate groups of animals increased in FST. ${ }^{15}$ After nephrotoxicity, neurotoxicity is the most severe CYA-related side effect that was proved to be related to drug interface with brain mitochondria. ${ }^{5}$

In the following experiment, Crt prevented CYA-induced despair behavior, as the immobility time was significantly lower than the CYA group. It has been proven that $\mathrm{Crt}$ could protect neurons against neurotoxic substances such as harmful excitatory amino acid glutamate levels by buffering ATP levels. ${ }^{24}$ The mitochondrion and $\mathrm{Crt}$ and $\mathrm{PCr}$ establish a critical system in energy homeostasis in high energy demanding organs, such as the brain. Crt buffers against ATP depletion since it increases $\mathrm{PCr}$ as a substrate for $\mathrm{Crt}$ kinase, which converts adenosine diphosphate to ATP and, therefore, exerts its neuroprotection effect. ${ }^{25}$ Therefore, by stimulating the rate of ATP synthesis and producing high amounts of $\mathrm{PCr}, \mathrm{Crt}$ may have prevented the CYA-induced neurotoxicity and depressive-like behavior in mice. Pretreatment with $\mathrm{Crt}$ also increased the swimming time, although neurotransmitters were not measured on the downside of the study; according to previous studies, the 
serotonergic system may be involved in the antidepressantlike effects. The SSRI drugs increase the swimming time while the catecholamine-related antidepressants increase the climbing time. ${ }^{26}$ However, the pretreatment of fluoxetine with CYA showed unexpected results as the climbing time was significantly higher than control because of the interaction between CYA and fluoxetine. It was shown previously that high dose CYA decreases serotonin release. ${ }^{6}$

Pretreatment with ALA also reduced CYA-induced depressionlike behavior, as immobility time was reduced significantly. ALA has been recognized as a potent antioxidant naturally found in diets; indeed, there would be increased functional capacity when given as a supplement. ${ }^{27}$ ALA antioxidant capacity could be the reason for its protective effects against CYA-induced depression. In support of our study, animal studies have shown that ALA, along with reducing neurodegeneration in the hippocampus, reduced peripheral oxidative damage by increasing total anti-oxidative potential. ${ }^{28}$ In addition, supplemented ALA in old rats has increased mitochondrial membrane capacity and declined oxidative damage. ${ }^{29}$ Therefore ALA has prevented CYA neurotoxicity through interaction with brain mitochondria functioning. ${ }^{5}$ The most exciting finding was that co-administrating ALA-Crt had a synergistic antidepressant-like effect as immobility time during FST reduced dramatically. Meanwhile, climbing and particularly swimming time significantly increased. It was supposed that serotonin and mitochondria have a close interconnection since by improving mitochondrial functioning, swimming time increased. Variable mitochondrial activity equals energy demand to energy supply throughout the neurons and controls replacing mitochondria in the periphery. ${ }^{30}$ Researchers have found that serotonin and 5-HT1A receptors in hippocampal neurons are involved in mitochondrial trafficking. ${ }^{31}$

In the present study, brain BDNF levels almost doubled following CYA administration, and AIA similar to fluoxetine prevented this effect. BDNF and its receptor tyrosine kinase receptor B play essential roles in cell survival, neurogenesis, synaptic plasticity, and neuron survival during life. ${ }^{32}$ Previous studies have shown that chronic administration of CYA in rats for 30 days reduced brain BDNF levels that could be responsible for the depressant effect of CY. ${ }^{8}$ It was observed that following 14 days of treatment with fluoxetine, BDNF expression was decreased. ${ }^{33}$ However, following 21 days of fluoxetine administration, BDNF expression was upregulated. ${ }^{34}$ It was also reported that BDNF level increased in an animal model of schizophrenia, and it was suggested that this elevation is in response to toxic materials as a defensive mechanism. ${ }^{35,36}$ After 7 days of BDNF infusions into the ventral tegmental area, latency to immobility in the FST declined, suggesting a depressive-like behavior against the BDNF role proposed in the hippocampus. ${ }^{37}$ In addition, some studies have shown that increased BDNF level is related to depression. ${ }^{38}$ According to these studies, different therapies and exposure time could influence brain BDNF levels. In our study, after depressive-like behavior initiated by CYA, BDNF levels increased, that might be related to CYA neurotoxic effect. However, although $\mathrm{Crt}$ pretreatment had an anti-immobility effect in FST, it did not alter the rise in BDNF levels. Only ALA pretreatment reduced BDNF levels similar to fluoxetine, and probably, ALA is more effective in preventing CYA neurotoxic initiation; this warrants further investigation. In addition, the higher hippocampus to whole-brain ratio and elevation of BDNF level in CYA treated groups are related to the increased immobility during FST.

\section{CONCLUSION}

In conclusion, the present study showed that mitochondrial antioxidant ALA and Crt prevented CYA-induced depressivelike behavior. Therefore, it was postulated that at least part of CYA induced depressive effect is mediated by mitochondrial dysfunction neurotoxicity. Also, we observed that ALA could hinder BDNF alteration and its possible neurological changes as effective as fluoxetine.

\section{ACKNOWLEDGEMENTS}

This work was supported by the School of Pharmacy and Pharmaceutical Sciences Research Council, Isfahan University of Medical Sciences (grant number 399189, 1/05/2020).

\section{Ethics}

Ethics Committee Approval: Care and Use of Laboratory Animals Guidelines Issued by The National Ethical Committee of Vice-Chancellor in Research Affairs-Medical University of Isfahan (ethical no: IR.MUI.RESEARCH.REC.1399.200).

Informed Consent: Not applicable.

Peer-review: Externally peer-reviewed.

\section{Authorship Contributions}

Concept: M.A., A.M., Design: A.M., M.A., Data Collection or Processing: A.S.M., A.M., Analysis or Interpretation: A.M., M.A., Literature Search: A.M., M.A., A.S.M., Writing: A.M., M.A.

Conflict of Interest: No conflict of interest was declared by the authors.

Financial Disclosure: This research was supported by the Research Council, Isfahan University of Medical Sciences, Iran.

\section{REFERENCES}

1. Buttini M, Limonta S, Luyten M, Boddeke $H$. Differential distribution of calcineurin A isoenzyme mRNA's in rat brain. Naunyn Schmiedebergs Arch Pharmacol. 1993;348:679-683.

2. Mansuy IM. Calcineurin in memory and bidirectional plasticity. Biochem Biophys Res Commun. 2003;311:1195-1208.

3. Musson RE, Cobbaert CM, Smit NP. Molecular diagnostics of calcineurin-related pathologies. Clin Chem. 2012;58:511-522.

4. Kahl AL, Kirchhof J, Füting A, Hütter BO, Wilde B, Witzke O, Benson S, Hadamitzky M, Schedlowski M. Acute administration of cyclosporine A does not impair attention or memory performance in healthy men. Behav Pharmacol. 2017;28:255-261.

5. Serkova NJ, Christians U, Benet LZ. Biochemical mechanisms of cyclosporine neurotoxicity. Mol Interv. 2004;4:97-107. 
6. Sato Y, Takayanagi Y, Onaka T, Kobayashi E. Impact of cyclosporine upon emotional and social behavior in mice. Transplantation. 2007;83:13651370.

7. Lewin GR, Barde YA. Physiology of the neurotrophins. Annu Rev Neurosci. 1996;19:289-317.

8. Chen CC, Hsu LW, Huang LT, Huang TL. Chronic administration of cyclosporine a changes expression of BDNF and TrkB in rat hippocampus and midbrain. Neurochem Res. 2010;35:1098-1104.

9. Pakzad D, Akbari V, Sepand MR, Aliomrani M. Risk of neurodegenerative disease due to tau phosphorylation changes and arsenic exposure via drinking water. Toxicol Res (Camb). 2021;10:325-333.

10. Andres RH, Ducray AD, Schlattner $U$, Wallimann T, Widmer HR. Functions and effects of creatine in the central nervous system. Brain Res Bull. 2008;76:329-343.

11. Nasrallah F, Feki M, Kaabachi N. Creatine and creatine deficiency syndromes: biochemical and clinical aspects. Pediatr Neurol. 2010;42:163-171.

12. Gualano B, Artioli GG, Poortmans JR, Lancha Junior AH. Exploring the therapeutic role of creatine supplementation. Amino Acids. 2010;38:3144.

13. Deng C, Sun Z, Tong G, Yi W, Ma L, Zhao B, Cheng L, Zhang J, Cao F, Yi $D . \alpha$-Lipoic acid reduces infarct size and preserves cardiac function in rat myocardial ischemia/reperfusion injury through activation of PI3K/ Akt/Nrf2 pathway. PLoS One. 2013;8:e58371.

14. Bist R, Bhatt DK. The evaluation of effect of alpha-lipoic acid and vitamin $\mathrm{E}$ on the lipid peroxidation, gamma-amino butyric acid and serotonin level in the brain of mice (Mus musculus) acutely intoxicated with lindane. J Neurol Sci. 2009;276:99-102.

15. Mesripour A, Golbidi M, Hajhashemi V. Dextromethorphan improved cyclosporine-induced depression in mice model of despair. Res Pharm Sci. 2020;15:447-453.

16. Alraddadi EA, Lillico R, Vennerstrom JL, Lakowski TM, Miller DW. Absolute oral bioavailability of creatine monohydrate in rats: debunking a myth. Pharmaceutics. 2018;10:31.

17. Zou J, Gan X, Zhou H, Chen X, Guo Y, Chen J, Yang X, Lei J. Alpha-lipoic acid attenuates cardiac hypertrophy via inhibition of $\mathrm{C} / \mathrm{EBP} \beta$ activation. Mol Cell Endocrinol. 2015;399:321-329.

18. Hemsley KM, Hopwood JJ. Development of motor deficits in a murine model of mucopolysaccharidosis type IIIA (MPS-IIIA). Behav Brain Res. 2005;158:191-199.

19. Azimi Fashi Y, Mesripour A, Hajhashemi V. Evaluation of the effect of soybean diet on interferon- $\alpha$-induced depression in male mice. Avicenna J Phytomed. 2017;7:436-443.

20. Mesripour A, Meshkati A, Hajhashemi V. A synbiotic mixture augmented the efficacy of doxepin, venlafaxine, and fluvoxamine in a mouse model of depression. Turk J Pharm Sci. 2020;17:293-298.

21. Paknejad B, Shirkhanloo H, Aliomrani M. Is there any relevance between serum heavy metal concentration and BBB leakage in multiple sclerosis patients? Biol Trace Elem Res. 2019;190:289-294.

22. Allen PJ, D'Anci KE, Kanarek RB, Renshaw PF. Chronic creatine supplementation alters depression-like behavior in rodents in a sexdependent manner. Neuropsychopharmacology. 2010;35:534-546.
23. Deussing JM. Animal models of depression. Drug Discovery Today: Disease Model. 2006;3:375-383.

24. Brustovetsky N, Brustovetsky T, Dubinsky JM. On the mechanisms of neuroprotection by creatine and phosphocreatine. J Neurochem. 2001;76:425-434.

25. Matthews RT, Ferrante RJ, Klivenyi P, Yang L, Klein AM, Mueller G, Kaddurah-Daouk R, Beal MF. Creatine and cyclocreatine attenuate MPTP neurotoxicity. Exp Neurol. 1999;157:142-149.

26. Cryan JF, Markou A, Lucki I. Assessing antidepressant activity in rodents: recent developments and future needs. Trends Pharmacol Sci. 2002;23:238-245.

27. Anthony RM, MacLeay JM, Gross KL. Alpha-lipoic acid as a nutritive supplement for humans and animals: an overview of its use in dog food. Animals (Basel). 2021;11:1454.

28. Cui X, Zuo P, Zhang Q, Li X, Hu Y, Long J, Packer L, Liu J. Chronic systemic $\mathrm{D}$-galactose exposure induces memory loss, neurodegeneration, and oxidative damage in mice: protective effects of R- $\alpha$-lipoic acid. $J$ Neurosci Res. 2006;83:1584-1590.

29. Liu J, Head E, Gharib AM, Yuan W, Ingersoll RT, Hagen TM, Cotman $\mathrm{CW}$, Ames BN. Memory loss in old rats is associated with brain mitochondrial decay and RNA/DNA oxidation: partial reversal by feeding acetyl-L-carnitine and/or R- $\alpha$-lipoic acid. Proc Natl Acad Sci U S A. 2002;99:2356-2361.

30. Schwarz TL. Mitochondrial trafficking in neurons. Cold Spring Harb Perspect Biol. 2013;5:a011304.

31. Chen S, Owens GC, Crossin KL, Edelman DB. Serotonin stimulates mitochondrial transport in hippocampal neurons. Mol Cell Neurosci. 2007;36:472-483.

32. Webster MJ, Herman MM, Kleinman JE, Shannon Weickert C. BDNF and trkB mRNA expression in the hippocampus and temporal cortex during the human lifespan. Gene Expr Patterns. 2006;6:941-951.

33. Miró X, Pérez-Torres $S$, Artigas $F$, Puigdomènech $P$, Palacios JM, Mengod G. Regulation of cAMP phosphodiesterase mRNAs expression in rat brain by acute and chronic fluoxetine treatment. An in situ hybridization study. Neuropharmacology. 2002;43:1148-1157.

34. De Foubert G, Carney SL, Robinson CS, Destexhe EJ, Tomlinson R, Hicks CA, Murray TK, Gaillard JP, Deville C, Xhenseval V, Thomas CE, O'Neill MJ, Zetterström TS. Fluoxetine-induced change in rat brain expression of brain-derived neurotrophic factor varies depending on length of treatment. Neuroscience. 2004;128:597-604.

35. Hirota K, Lambert DG. Ketamine: its mechanism(s) of action and unusual clinical uses. Br J Anaesth. 1996;77:441-444.

36. Guo C, Yang Y, Su Y, Si T. Postnatal BDNF expression profiles in prefrontal cortex and hippocampus of a rat schizophrenia model induced by MK-801 administration. J Biomed Biotechnol. 2010;2010:783297.

37. Eisch AJ, Bolaños CA, de Wit J, Simonak RD, Pudiak CM, Barrot M, Verhaagen J, Nestler EJ. Brain-derived neurotrophic factor in the ventral midbrain-nucleus accumbens pathway: a role in depression. Biol Psychiatry. 2003;54:994-1005.

38. Branchi I, D'Andrea I, Sietzema J, Fiore M, Di Fausto V, Aloe L, Alleva E. Early social enrichment augments adult hippocampal BDNF levels and survival of BrdU-positive cells while increasing anxiety- and “depression”-like behavior. J Neurosci Res. 2006;83:965-973. 